\title{
Stability of the homology of the moduli spaces of Riemann surfaces with spin structure
}

\author{
John L. Harer *
}

Department of Mathematics, University of Michigan, Ann Arbor, MI 48109, USA

\section{Introduction}

Recently, due largely to its importance in fermionic string theory, there has been much interest in the moduli spaces $\mathscr{M}_{g}[\varepsilon]$ of Riemann surfaces of genus $g$ with spin structure of Arf invariant $\varepsilon \in \mathbb{Z} / 2 \mathbb{Z}$. Algebraic geometers have long studied these spaces in their alternate guise as moduli spaces of pairs (algebraic curve, square root of the canonical bundle). For topologists these spaces are rational classifying spaces for spin mapping class groups. However, despite the fact that $\mathscr{M}_{g}[\varepsilon]$ is a finite cover of the ordinary moduli space $\mathscr{H}_{g}$, little is known about the topology of these spaces.

In this paper we begin a study of the homology of $\mathscr{M}_{g}[\varepsilon]$ by proving that its homology groups are independent of $g$ and $\varepsilon$ when $g$ is adequately large (Theorem 3.1). In a second paper [H4] we will compute $H_{1}\left(\mathscr{M}_{g}[\varepsilon]\right)$ and $H_{2}\left(\mathscr{H}_{g}[\varepsilon] ; \mathbb{Q}\right)$, thereby calculating the Picard group of $\mathscr{M}_{g}[\varepsilon]$. Putting this all together we know approximately the same amount about the homology of $\mathscr{M}_{g}[\varepsilon]$ as we do about that of $\mathscr{M}_{g}$ itself.

The techniques used here are an extension of those of [ $\left.\mathrm{H}_{2} 2\right]$ which are in turn strongly related to those of $[\mathrm{C} ; \mathrm{Q} ; \mathrm{V} ; \mathrm{W}]$ and others. We begin by constructing several simplicial complexes from configurations of simple closed curves and properly imbedded arcs in a surface of genus $g$. The homology of the spin moduli space is identified with that of the spin mapping class group $G$, which acts on these complexes in a natural way. The Borel construction is then applied to obtain a spectral sequence which describes the homology of $G$ in terms the homology of the stabilizers of the cells of these complexes. These turn out to be spin mapping class groups (in an extended sense) of smaller genus and the result is established inductively. The complexes are exactly the same as those of [H 2]; however, the spectral sequence arguments are more difficult because there are more orbits of cells under the action of $G$. Furthermore, in Sect. 4 we apply an entirely different and much simpler version of the argument of [H 2] to obtain stability in the case of a closed surface.

\footnotetext{
* This work was supported by grants from the Sloan Foundation, the National Science
} Foundation and the CNR 


\section{Mod 2 quadratic forms, arc systems, loop systems and curve systems}

Let $F=F_{g, 1}$ be a smooth orientable surface of genus $g$ with one boundary component. $\mathrm{A} \mathbb{Z} / 2 \mathbb{Z}$-quadratic form on the $\mathbb{Z} / 2 \mathbb{Z}$ vector space $V=H_{1}(F ; \mathbb{Z} / 2 \mathbb{Z})$ is a $\operatorname{map} Q: V \rightarrow \mathbb{Z} / 2 \mathbb{Z}$ such that for every $x, y$ in $V, Q(x+y)=Q(x)+Q(y)+x \cdot y$, where $x \cdot y$ is the $\bmod 2$ intersection number of $x$ and $y$. Given $g$ the possible $Q$ are classified up to isomorphism by the Arf invariant $\varepsilon=\varepsilon(Q)=0$ or 1 (see, e.g., [RS]). If $\left\{x_{i}, y_{i} ; 1 \leqq i \leqq g\right\}$ is a symplectic basis of $V$, i.e.:

$$
x_{i} \cdot x_{j}=y_{i} \cdot y_{j}=0, \quad x_{i} \cdot y_{j}=\delta_{i j}, \text { for all } i, j,
$$

then

$$
\varepsilon=Q\left(x_{1}\right) \cdot Q\left(y_{1}\right)+\ldots+Q\left(x_{g}\right) \cdot Q\left(y_{g}\right) .
$$

Note that the form $Q$ assigns a 0 or a 1 to every unoriented simple closed curve $C$ in $F$ by evaluating $Q$ on the mod 2 homology class represented by $C$.

If $F=F_{g, 1}$ is included in $F_{g+1,1}$, inducing an inclusion on homology groups $V_{g} \rightarrow V_{g+1}$, then $V_{g+1}$ may be given a quadratic form $Q_{g+1}$ of arbitrary Arf invariant extending $Q=Q_{g}$ by setting $Q_{g+1}\left(x_{g+1}\right)=Q_{g+1}\left(y_{g+1}\right)=0$ or 1 . This elementary observation is at the heart of the fact that the stable homology of $M_{g}[\varepsilon]$ is independent of $\varepsilon$.

Suppose now that $F_{g, r}$ is a smooth oriented surface of genus $g$ with $r$ boundary components. The mapping class group $\Gamma_{g, r}=\Gamma\left(F_{g, r}\right)$ is $\pi_{0}\left(\operatorname{Diff}^{+}\left(F_{g, r}, \partial\right)\right)$ where $\operatorname{Diff}^{+}\left(F_{g, r} \partial\right)$ is the group of orientation preserving diffeomorphisms of $F_{g, r}$ which restrict to the identity on $\partial=\partial F_{g, r}$. The group $\Gamma_{g, r}$ acts properly discontinuously (and freely when $r>0$ ) on the Teichmüller space $\mathscr{T}_{g, r}$ of marked Riemann surfaces $X$ with $r$ pairs $\left(p_{i}, v_{i}\right), p_{i}$ a point of $X$ and $v_{i}$ a nonzero tangent vector to $X$ at $p_{i}$. The quotient $\mathscr{T}_{g, r} / \Gamma_{g, r}$ is moduli space $\mathscr{M}_{g, r}$. Since Teichmüller space is contractible, the rational homology of $\mathscr{M}_{g, r}$ and $\Gamma_{g, r}$ are the same.

Let $r=1$ and let $Q$ be a $\mathbb{Z} / 2 \mathbb{Z}$ quadratic form on $V$. Define $G=G(Q)=G_{g, 1}(Q)$ to be the subgroup of $\Gamma_{g, 1}$ that preserves $Q$; we call $G$ the spin mapping class group (we refer the reader to [LMW] and [E.B.] for an explanation of the relationship between $\mathbb{Z} / 2 \mathbb{Z}$ quadratic forms and spin structures on manifolds). The group $G$ contains the Dehn twist on any curve $C$ with $Q(C)=1$ and the square of the Dehn twist on any curve $C$ with $Q(C)=0$. The quotient of $\mathscr{T}_{g, r}$ by $G$ is one of the spin moduli spaces; there are two of these, determined up to isomorphism by the Arf invariant, and we denote them $\mathscr{M}_{g, 1}[0]$ and $\mathscr{M}_{g, 1}[1]$. Once again the fact that Teichmüller space is contractible implies that the rational homology of the spin mapping class groups is the same as that of the spin moduli spaces. We will be studying the behavior of the homology of the groups $G_{g, 1}(Q)$ as $g$ gets large.

We recall now the loop-system, arc-system and curve-system complexes of [H 2] and [H 3]. Let $F=F_{g, r}$ and let $p$ be a point on $\partial_{1} F$. A loop system $\left\langle\alpha_{0}, \ldots, \alpha_{k}\right\rangle$ is the isotopy class rel $p$ of a family of embedded loops $\left\{\alpha_{0}, \ldots, \alpha_{k}\right\}$ based at $p$ such that for each $i \neq j$,

a) $\alpha_{i}$ and $\alpha_{j}$ intersect only at $p$,

b) $\alpha_{i}$ is not homotopic to a point and

c) $\alpha_{i}$ is not homotopic rel $p$ to $\alpha_{j}$. 
Form a simplicial complex $A X$ by taking a $k$-simplex for each $\left\langle\alpha_{0}, \ldots, \alpha_{k}\right\rangle$ such that $F-\left\{\alpha_{i}\right\}$ is connected and identifying $\left\langle\alpha_{0}, \ldots, \alpha_{k}\right\rangle$ as a face of $\left\langle\alpha_{0}^{\prime}, \ldots, \alpha_{1}^{\prime}\right\rangle$ when there are representatives of the isotopy classes such that $\left\{\alpha_{i}\right\} \subset\left\{\alpha_{j}^{\prime}\right\}$. The mapping class group $\Gamma_{g, r}$ acts on $A X$ in the obvious way. (Remark: If $\alpha=\left\langle\alpha_{0}, \ldots, \alpha_{k}\right\rangle$ we will use the letter $\alpha$ interchangeably to denote the simplex, the loop system and the elements of the set $\left\{\alpha_{0}, \ldots, \alpha_{k}\right\}$.)

We will need a second complex $B X$ defined as follows. Let $r \geqq 2$ and let $p_{i}$ be a point on $\partial_{i} F, i=1,2$. An arc system $\left\langle\beta_{0}, \ldots, \beta_{k}\right\rangle$ is the isotopy class of a family of properly imbedded arcs $\left\{\beta_{0}, \ldots, \beta_{k}\right\}$ connecting $p_{1}$ to $p_{2}$ such that for each $i \neq j$,

a) $\beta_{i}$ and $\beta_{j}$ intersect only at $p_{1}$ and $p_{2}$ and

b) $\beta_{i}$ is not homotopic to $\beta_{j} \operatorname{rel}\left\{p_{1}, p_{2}\right\}$.

The simplicial complex $B X$ is defined exactly like $A X$ using arc systems which do not disconnect $F$. Again $\Gamma_{g, r}$ acts on $B X$ in the obvious way.

Finally, a third simplicial complex $X$ is defined as follows. A curve system $C=\left\langle C_{0}, \ldots, C_{k}\right\rangle$ is the isotopy class of a family of disjoint simple closed curves in $F$ such that for each $i \neq j$ :

a) $C_{i}$ is not homotopic to a point and

b) $C_{i}$ is not homotopic to $C_{j}$.

The simplicial complex $X$ is constructed in the same way as were $A X$ and $B X$, using curve-systems which do not separate $F$. Once again $\Gamma_{g, r}$ acts on $X$ in the obvious way.

An $n$-dimensional simplicial complex is called spherical if it is homotopy equivalent to a wedge of spheres of dimension $n$. The following lemma was proven is [H 2].

Lemma 1.1. a) $A X$ is spherical of dimension $2 g-1$.

b) $B X$ is spherical of dimension $2 \mathrm{~g}$.

c) $X$ is spherical of dimension $\mathrm{g}-1$.

If $G$ is any subgroup of $\Gamma_{g, r}$, then $G$ also acts on $A X$ and $B X$ so that they may be used to study the homology of $G$. Being specific, the Borel construction (see, e.g. [K.B.]) gives us an augmented homology spectral sequence $\left(E^{*}, d^{*}\right)$ converging to 0 for $p+q<$ the dimension of $A X, B X$ or $X$ respectively with $E^{1}$ term constructed from the homology of the stabilizers of the simplices and the homology of $G$. More precisely, if $p \geqq 0$

$$
E_{p, q}^{1}=\oplus_{\sigma_{p}} H_{q}\left(G_{\sigma_{p}}\right),
$$

where the sum is over representatives of the orbits of the $p$-cells under the action of $G$ and $G_{a_{p}}$ denotes the stabilizer of $\sigma_{p}$. The augmentation gives $E_{-1, q}^{1}=H_{q}(G)$.

We will use these spectral sequences in Sects. 3 and 4 to study $H_{*}(G)$.

\section{Description of the action on $A X, B X$, and $X$}

Let $F$ be a surface of genus $g_{0} \geqq 1$ with one boundary component and let $Q$ be a $\mathbb{Z} / 2 \mathbb{Z}$-quadratic form on $V=H_{1}(F ; \mathbb{Z} / 2 \mathbb{Z})$ of arf invariant $\varepsilon$. Fix a point $p$ on $\partial F$ and let $\gamma=\left\langle\gamma_{0}, \ldots, \gamma_{n}\right\rangle$ be a loop system based at $p$ with $F-\left\{\gamma_{i}\right\}$ connected. Write 
$\Gamma(\gamma)$ for the stabilizer of $\gamma$ in $\Gamma_{g, 1}, G(Q)$ for the stabilizer of $Q$ in $\Gamma$ and $G(\gamma)=G(Q)$ $\cap \Gamma(\gamma)$. If $F(\gamma)$ is the surface obtained by splitting $F$ open along $\gamma, \Gamma(\gamma)$ may be identified with the mapping class group $\Gamma_{g, r}$, where $F(\gamma)$ has genus $g$ and $r$ boundary components. $G(\gamma)$ is then identified with a subgroup of $\Gamma_{g, r}$.

In order to study $H_{*}(G(\gamma))$ we will need to describe the orbits of the action of $G(\gamma)<\Gamma_{g, r}$ on the complexes $A X, B X$, and $X$. Here these complexes are constructed using loop, arc and curve systems on $F(\gamma)$ and we will always assume that the points $p_{1}$ and $p_{2}$ are chosen from the collection of points which become identified to $p$ if we reglue $F(\gamma)$ to obtain $F$. In this way each loop/arc system $\alpha$ in $A X$ or $B X$ gives rise to a loop system $\langle\gamma, \alpha\rangle$ in $F$. Furthermore, each loop/arc/curve has a $Q$ value of 0 or 1 which must be preserved by $G(\gamma)$. We need now to describe the orbits of the action of $G(\gamma)$.

Consider $A X$ first. Fix $k \geqq 0$ and let $\Omega_{k}$ be the set of all unordered groupings $\left(\begin{array}{ll}1 & i_{2}\end{array}\right)\left(i_{3} i_{4}\right) \ldots\left(i_{2 k+1} i_{2 k+2}\right)$ of the elements of $\{1,2, \ldots, 2 k+2\}$ into pairs. Clearly $\Omega_{k}$ is finite (its order is $\left.(2 k+1) ! !=(2 k+1)(2 k-1) \ldots(3)(1)\right)$. Each loop system $\alpha=\left\langle\alpha_{0}, \ldots, \alpha_{k}\right\rangle$ determines an element $P(\alpha)$ in $\Omega_{k}$ (see [H 2], [H 3]): in a small neighborhood of $p$ there are $2 k+2$ segments emerging from $p$, number them consecutively and pair the numbers corresponding to the ends of each arc. In [H 2] we showed that for $k<g$ the association of $P(\alpha)$ to $\alpha$ gives a $1-1$ correspondence between the orbits of the $k$-cells of $A X$ under $\Gamma_{g, r}$ and the elements of $\Omega_{k}$. However, since $G(\gamma)$ is smaller there will be more orbits. In particular each $\alpha_{i}$ has a $Q$ value which must be preserved by $G(\gamma)$ so we will also need to associate to $\alpha$ the $k+1$ tuple $Q(\alpha)=\left(Q\left(\alpha_{0}\right), \ldots, Q\left(\alpha_{k}\right)\right)$ in $(\mathbb{Z} / 2 \mathbb{Z})^{k+1}$.

Lemma 2.1. Two $k$-cells $\alpha$ and $\alpha^{\prime}$ of $A X$ are equivalent under the action of $G(\gamma)$ if and only if $P(\alpha)=P\left(\alpha^{\prime}\right)$ and $Q(\alpha)=Q\left(\alpha^{\prime}\right)$. When $k<g-1$ all elements of $\Omega_{k} \times(\mathbb{Z} / 2 \mathbb{Z})^{k+1}$ occur.

Proof. Clearly if there exists an $f$ in $G(\gamma)$ such that $f(\alpha)=\alpha^{\prime}$ we must have $P(\alpha)$ $=P\left(\alpha^{\prime}\right)$ and $Q(\alpha)=Q\left(\alpha^{\prime}\right)$. Conversely, suppose $P(\alpha)=P\left(\alpha^{\prime}\right)$ and $Q(\alpha)=Q\left(\alpha^{\prime}\right)$. Since $\alpha$ and $\alpha^{\prime}$ are nonseparating $P(\alpha)=P\left(\alpha^{\prime}\right)$ implies that there is an $f_{0}$ in $\Gamma(\gamma)$ with $f_{0}(\alpha)=\alpha^{\prime}$. Because $Q(\alpha)=Q\left(\alpha^{\prime}\right) f_{0}$ preserves the $Q$ value of the loops in $\gamma$ and $\alpha$. Complete $\gamma \cup \alpha$ to a maximal loop system $\gamma \cup \alpha \cup \beta$ in $F$, it then consists of $2 g$ curves which form a basis for $H_{1} F$. If $Q\left(f_{0}\left(\beta_{i}\right)\right)=Q\left(\beta_{i}\right)$ for all $i, f_{0}$ lies in $G(\gamma)$. Otherwise we will do induction on $s$, the order of $\beta$, to show that $f_{0}$ may be altered to create $f$ as required.

Let $A=\alpha \cup \gamma, F_{0}=F-A$. If $s=1 F_{0}$ is an annulus with core circle $C$ intersecting $\beta_{1}$ in a single point. If $Q(C)=0$ and $Q\left(f_{0}\left(\beta_{1}\right)\right) \neq Q\left(\beta_{1}\right)$ form $f=f_{0} \tau_{C}\left(\tau_{C}\right.$ is the Dehn twist on $C)$. Then $Q\left(\tau_{c}\left(\beta_{1}\right)\right)=Q\left(\beta_{1}\right)+1$ so $f$ is the required map. If $Q\left(f_{0}(C)\right)=0$, forming $f=\tau_{f_{0}(C)} f_{0}$ also works, thus we may assume that $Q(C)=Q\left(f_{0}(C)\right)=1$. Now $\left\langle\beta_{1}, C\right\rangle$ is a direct summand $V_{0}$ of $V=H_{1}(F)$ as is $\left\langle f_{0}\left(\beta_{1}\right), f_{0}(C)\right\rangle$. Certain linear combinations of elements of $\Lambda$ with $C$ form an orthogonal basis to $\left\{\beta_{1}, C\right\}$ and provide us with orthogonal decompositions $V=V_{0} \oplus V_{1}=f_{0}\left(V_{0}\right) \oplus f_{0}\left(V_{1}\right)$. Since $f_{0}$ fixes the arf invariant of $V_{1}$ it must do so for $V_{0}$ and as $Q(C)=Q\left(f_{0}(C)\right)=1$ it follows that $Q\left(\beta_{1}\right)=Q\left(f_{0}\left(\beta_{1}\right)\right)$. This means that $f=f_{0}$ is the desired map.

When $s>1$ and $Q\left(\beta_{i}\right)=Q\left(f_{0}\left(\beta_{i}\right)\right)$ for some $i$ we incorporate $\beta_{i}$ into $A$ and induct. If there is a simple closed curve $C$ in $F_{0}$ with $Q(C)=0$ and $C \cap \beta_{i}$ equal to one point for some $i$ we replace $f_{0}$ by $f_{0} \tau_{c}$ to fix $Q\left(\beta_{i}\right)$ and induct. Thus we may assume that all 
curves $C$ in $F_{0}$ which meet any $\beta_{i}$ in one point have $Q$ value 1 . It is easy to see that this implies that $F_{0}$ is a punctured torus, so that $s=2$, with $Q\left(\beta_{i}\right)=1, i=1,2$. Also, $f_{0}\left(F_{0}\right)$ is a punctured torus, and if $Q\left(f_{0}\left(\beta_{i}\right)\right)=0$ we may form $f_{1}=\tau_{c} f_{0}$ where $C$ is the nonseparating closed curve which is the core of the annulus $f_{0}\left(F_{0}-\beta_{i}\right)$. This leaves only the possibility that $Q\left(f_{0}\left(\beta_{i}\right)\right)=1, i=1,2$ in which case $f_{0}$ lies in $G(\gamma)$.

Suppose now that $k<g-1$. Choose any element of $Q_{k} \times(\mathbb{Z} / 2 \mathbb{Z})^{k+1}$ and let $\alpha=\left\langle\alpha_{0}, \ldots, \alpha_{k}\right\rangle$ realize the element of $\Omega_{k}(\alpha$ exists for any $k<g$, see [H 2] for an argument). It is not hard to see that for each $i$ there exists a simple closed curve $C_{i}$ in $F-\gamma$ such that $C_{i}$ intersects $\alpha_{j}$ in $\delta_{i j}$ points and $Q\left(C_{i}\right)=0$ (this is where we use $k<g-1)$. If the $Q$ value of $\alpha_{i}$ is wrong replace it by $\tau_{C_{i}}\left(\alpha_{i}\right)$ and rechoose the other $C_{j}$. This completes the proof of 2.1.

Next we look at $B X$. Each arc-system $\beta$ determines an element $P(\beta)$ in the symmetric group $\Sigma_{k+1}$ as follows. Order the arcs counterclockwise as they emerge from $\partial_{1}$, the order that they encounter $\partial_{2}$ (measured clockwise) gives $P(\beta)$. As for loop systems each arc-system $\beta=\left\langle\beta_{0}, \ldots, \beta_{k}\right\rangle$ has a $Q$ value $Q(\beta)=\left(Q\left(\beta_{0}\right), \ldots, Q\left(\beta_{k}\right)\right)$ in $(\mathbb{Z} / 2 \mathbb{Z})^{k+1}$. The proof of Lemma 2.1 is easily adapted to show

Lemma 2.2. Two $k$-cells $\beta, \beta^{\prime}$ of $B X$ are equivalent under the action of $G(\gamma)$ if and only if $P(\beta)=P\left(\beta^{\prime}\right)$ and $Q(\beta)=Q\left(\beta^{\prime}\right)$. When $k<\mathrm{g}$ all elements of $\Sigma_{k+1} \times(\mathbb{Z} / 2 \mathbb{Z})^{k+1}$ are realized.

Finally, we look at $X$. Each curve system $C=\left\langle C_{0}, \ldots, C_{k}\right\rangle$ has a $Q$-value but, unlike the $A X$ and $B X$ cases, when 2 curves have the same $Q$-value they are permuted by an element of $G(\gamma)$ which fixes the other curves in the system. The only invariant then is $N(C)$ which we define to be the number of $C_{i}$ such that $Q\left(C_{i}\right)=1$. Once again the above techniques adapt easily to show:

Lemma 2.3. Two k-cells $C, C^{\prime}$ of $X$ are equivalent under the action of $G(\gamma)$ if and only if $N(C)=N\left(C^{\prime}\right)$.

When $k<\mathrm{g}-1$ all possibilities $0 \leqq N(C) \leqq k$ occur.

\section{Proof of stability in the bounded case}

In this section we will prove our main result that the homology of the groups $G(\gamma)$ is stable.

Suppose now that $\gamma^{\prime}$ is a loop system obtained from $\gamma$ by adding another loop $\alpha$. The stabilizer $G\left(\gamma^{\prime}\right)$ includes naturally in the group $G(\gamma)$. We will need to distinguish four cases:

Case 1. $\alpha$ connects two components $\partial_{1}$ and $\partial_{2}$ of $\partial F(\gamma)$ with $Q\left(\partial_{1}\right)$ and/or $Q\left(\partial_{2}\right)=0$ $\left(Q\left(\partial_{i}\right)\right.$ is the $Q$ value of a closed curve in the interior of $F(\gamma)$ homotopic to $\left.\partial_{i}\right)$.

Case 2. $\alpha$ is a loop in $F(\gamma)$ with $Q(\alpha)=1$,

Case 3. same as case 1 with $Q\left(\partial_{1}\right)=Q\left(\partial_{2}\right)=1$,

Case 4. $\alpha$ is a loop in $F(\gamma)$ with $Q(\alpha)=0$.

Theorem 3.1. The inclusion $G\left(\gamma^{\prime}\right) \rightarrow G(\gamma)$ induces

$$
H_{k}\left(G\left(\gamma^{\prime}\right)\right) \rightarrow H_{k}(G(\gamma))
$$


which is a surjection for:

a) $g \geqq 4 k-2$ in case 1 ,

b) $g \geqq 4 k-1$ in cases 2 and 3 ,

c) $g \geqq 4 k$ in case 4 ,

and an isomorphism for

a) $g \geqq 4 k-2$ in case 1 ,

b) $g \geqq 4 k+1$ in cases 2 and 3 ,

c) $g \geqq 4 k+2$ in case 4 .

Corollary 3.2. $H_{k}\left(\mathscr{M}_{g, 1}[Q]\right)$ is independent of $g$ and $Q$ for $g \geqq 4 k+1$.

To prove Theorem 3.1 we will make use of the spectral sequences associated to the action of $G(\gamma)$. For $p<g-1$ the spectral sequence for $A X$ has $E_{p, 0}^{1}$ equal to the free abelian group on the elements of $\Omega_{p} \times(\mathbb{Z} / 2 \mathbb{Z})^{p+1}$ and (using freely the correspondence between these elements and orbits of loop systems)

$$
d_{p, 0}^{1}\left(\left\langle\alpha_{0}, \ldots, \alpha_{k}\right\rangle\right)=\sum_{i=0}^{p}(-1)^{i} \varphi_{i}\left(\left\langle\alpha_{0}, \ldots, \hat{\alpha}_{i}, \ldots, \alpha_{k}\right\rangle\right)
$$

where $\varphi_{i}$ identifies $\left\langle\alpha_{0}, \ldots, \hat{\alpha}_{i}, \ldots, \alpha_{k}\right\rangle$ with the representative of its orbit. Define

$$
D_{A}: E_{p, 0}^{1} \rightarrow E_{p+1,0}^{1}
$$

by

$$
\begin{gathered}
D_{A}\left(\left(\begin{array}{ll}
1 & i_{2}
\end{array}\right)\left(i_{3} i_{4}\right) \ldots\left(i_{2 p+1} i_{2 p+2}\right),\left(\delta_{0}, \ldots, \delta_{p}\right)\right) \\
=\left((1 \quad 2)\left(3 \quad i_{2}+2\right) \ldots\left(i_{2 p+1}+2 i_{2 p+2}+2\right),\left(1, \delta_{0}, \ldots, \delta_{p}\right)\right) .
\end{gathered}
$$

Then $D_{A} d+d D_{A}=1$. It is easy to show that all elements of $\Omega_{p} \times(\mathbb{Z} / 2 \mathbb{Z})^{p+2}$ with first $Q$ value 1 exist when $g \geqq p+2$, so $E_{p, 0}^{2}=0$ for $p \leqq g-2$.

Similarly for $B X, E_{p, 0}^{1}$ is the free abelian group on the elements of $\Sigma_{p+1}$ $\times(\mathbb{Z} / 2 \mathbb{Z})^{p+1}, p<g$, with the same formula for $d_{p, 0}^{1}$. Define

$$
D_{B}: E_{p, 0}^{1} \rightarrow E_{p+1,0}^{1}
$$

by

$$
D_{B}\left(\sigma,\left(\delta_{0}, \ldots, \delta_{p}\right)\right)=\left(\lambda(\sigma),\left(1, \delta_{0}, \ldots, \delta_{p}\right)\right),
$$

where $\lambda$ is the inclusion of $\Sigma_{p+1}$ into $\Sigma_{p+2}$ as permutations of $(2, \ldots, p+2\}$. Again $D_{B} d+d D_{B}=1$ and all elements of $\Sigma_{p+1} \times(\mathbb{Z} / 2 \mathbb{Z})^{p+2}$ with first $Q$ value 1 exist when $g \geqq p+1$, so $E_{p, 0}^{2}=0$ for $p \leqq g-2$.

For $q>0$,

$$
\left.d_{p, q}^{1}\right|_{H_{q}\left(G_{\left.\alpha^{\prime}\right)}\right.}=\sum_{\alpha \text { a face of } \alpha^{\prime}} \pm\left(\varphi_{\alpha} \chi_{\left(\alpha^{\prime}, \alpha\right)}\right)_{*}
$$

where

$$
\chi_{\left(\alpha^{\prime}, \alpha\right)}: G_{\alpha^{\prime}} \rightarrow G_{\alpha}
$$

is the inclusion and

$$
\varphi_{\alpha}: G_{\alpha} \rightarrow G_{\alpha_{0}}
$$


is induced by the identification under $G(\gamma)$ of $\alpha$ with the appropriate orbit representative $\alpha_{0}$ ([Brown]). The important thing to notice here is that each map $\chi_{\left(\alpha^{\prime}, a\right)}$ may be identified as an inclusion $G_{\gamma^{\prime}} \rightarrow G_{\gamma}$ so that Theorem 3.1 applies to it as well.

We are now in a position to prove 3.1 inductively, so assume it is known for all homology groups of dimension less than $k$. The proof has two parts, each with a lemma and its application to the four cases.

\section{Part 1. Surjectivity}

Using $A X$ and $B X$ we obtain two spectral sequences.

Lemma 3.3. The map

$$
d_{0, k}^{1}: E_{0, k}^{1} \rightarrow E_{-1, k}^{1}=H_{k}(G(\gamma))
$$

is surjective for the $A X$ spectral sequence when $g \geqq 4 k-1$, and for the $B X$ spectral sequence when $g \geqq 4 k-2$.

Proof. First look at $A X$. For each loop system $\alpha=\left\langle\alpha_{0}, \ldots, \alpha_{p}\right\rangle$, we have genus $(F(\gamma$ $\cup \alpha)) \geqq g-(p+1)$. If $q<k$, and $\alpha$ is a face of $\alpha^{\prime}$, then

$$
\chi_{\left(\alpha^{\prime}, \alpha\right)}: G_{\alpha^{\prime}} \rightarrow G_{\alpha}
$$

induces an isomorphism on $H_{q}$ when $g-(p+1) \geqq 4 q+2$ and a surjection when $g-(p+1) \geqq 4 q$. Furthermore, if $\alpha_{1}$ and $\alpha_{2}$ are rank $p$ faces of $\alpha^{\prime}$ which are identified by $G(\gamma)$, say $\lambda\left(\alpha_{1}\right)=\alpha_{2}$, then we claim that $\lambda \cdot \chi_{\left(\alpha^{\prime}, a_{1}\right)}$ induces the same map as does $\chi_{\left(\alpha^{\prime}, \alpha_{2}\right)}$ for $g-(p+2) \geqq 4 q-1$. To see this, let $F\left(\gamma \cup \alpha^{\prime}\right)$ have genus $g_{0} \geqq g-(p+2)$. Then there exists a subsurface $F_{0} \subset F\left(\gamma \cup \alpha^{\prime}\right)$ of genus $g_{0}$ with $r$ boundary components (the same number as $F(\gamma)$ ) such that $\alpha^{\prime}$ is completely outside $F_{0}$. The map $\lambda$ may now be chosen to fix $F_{0}$. By out inductive assumption

$$
H_{q}\left(G\left(F_{0}\right)\right) \rightarrow H_{q}\left(G\left(\gamma \cup \alpha^{\prime}\right)\right)
$$

is surjective for $g_{0} \geqq 4 q-1$, establishing the claim.

Now, when $k>p+q$ and $g \geqq 4 k-1$, we have

$$
g \geqq 4 k-1 \geqq 4 p+4 q+3 \geqq p+4 q+3
$$

which implies $E_{p, q}^{2}=0$. When $k=p+q, q<k$ and $g \geqq 4 k-1$, we have

$$
g \geqq 4 p+4 q-1>4 q+p+1
$$

(since $p \geqq 1$ ), so $E_{p, q}^{2}=0$ again. The lemma now follows for $A X$.

For $B X$ if $\beta=\left\langle\beta_{0}, \ldots, \beta_{k}\right\rangle$ is an arc system then genus $(F(\gamma \cup \beta)) \geqq g-p$. The argument is now the same as for $A X$ except for the adjustment of $g$ by 1 . 4.

Next we use this lemma to establish surjectivity of the map on $H_{k}$ for Cases 1 to

Case 1. The $B X$ case of Lemma 3.3 gives us that when $g \geqq 4 k-2$ and $\gamma_{i}$ is obtained from $\gamma$ by adding an arc $\beta_{i}$ connecting $\partial_{1}$ with $\partial_{2}$ with $Q\left(\beta_{i}\right)=i$, then

$$
H_{k}\left(G\left(\gamma_{0}\right)\right) \oplus H_{k}\left(G\left(\gamma_{1}\right)\right) \rightarrow H_{k}(G(\gamma))
$$


is surjective. Suppose that labels are chosen so that $Q\left(\partial_{1}\right)=0$. We may assume that $\beta_{1}=\tau\left(\beta_{0}\right)$ where $\tau$ is the Dehn twist on a curve parallel to $\partial_{1}$. However, it is immediate that $\tau^{(-1)^{i}}$ acts trivially on the image of $H_{k}\left(G\left(\gamma_{i}\right)\right)$ in $H_{k}(G(\gamma))$, so surjectivity is established in Case 1.

Case 2. The $A X$ case of Lemma 3.3 gives us that when $g \geqq 4 k-1$ and $\gamma_{i}$ is obtained from $\gamma$ by adding a loop $\alpha_{i}$ with $Q$ value $i$, then

$$
H_{k}\left(G\left(\gamma_{0}\right)\right) \oplus H_{k}\left(G\left(\gamma_{1}\right)\right) \rightarrow H_{k}(G(\gamma))
$$

is surjective. We may assume that $\alpha_{0}$ and $\alpha_{1}$ are chosen so that together they form an edge $\alpha=\left\langle\alpha_{0}, \alpha_{1}\right\rangle$ with $P(\alpha)=\left(\begin{array}{lll}1 & 3\end{array}\right)\left(\begin{array}{ll}2 & 4\end{array}\right)$. Then the map

$$
H_{k}(G(\gamma \cup \alpha)) \rightarrow H_{k}\left(G\left(\gamma_{0}\right)\right)
$$

is surjective for $g-1 \geqq 4 k-2$ by case 1 . This means that

$$
H_{k}\left(G\left(\gamma_{1}\right)\right) \rightarrow H_{k}(G(\gamma))
$$

is surjective which is Case 2 .

Case 3. Look again at $B X$; as before 3.3 gives $\left(*_{1}\right)$ surjective for $g \geqq 4 k-2$. We may choose the $\beta_{i}$ so that together they form an edge $\beta=\left\langle\beta_{0}, \beta_{1}\right\rangle$ with $P(\beta)$ equal to the identity permutation in $\Sigma_{2}$. Then the map

$$
H_{k}(G(\gamma \cup \beta)) \rightarrow H_{k}\left(G\left(\gamma_{i}\right)\right)
$$

is surjective for $g \geqq 4 k-1$ by Case 2 , either $i$. This proves Case 3 .

Case 4. Finally, we return to $A X$ where $\left(*_{2}\right)$ is surjective for $g \geqq 4 k-1$. Case 3 applies to show

$$
H_{k}(G(\gamma \cup \alpha)) \rightarrow H_{k}\left(G\left(v_{1}\right)\right)
$$

is surjective when $g-1 \geqq 4 k-1$ ( $\alpha$ as in Case 2). This establishes Case 4 .

\section{Part 2. Injectivity}

Case 1 does not require the spectral sequence argument. If (say) $Q\left(\partial_{1}\right)=0$, then we may attach a disk to $\partial_{1}$ and extend by the identity to define

$$
G\left(\gamma^{\prime}\right) \stackrel{\xi}{\longrightarrow} G(\gamma)
$$

such that $\xi \cdot \chi=1$ ( $\chi$ the inclusion). This means that $\chi_{*}$ is injective on $H_{k}$ for every $g$. Note that when $Q\left(\partial_{1}\right)=1$ this map is defined but it maps $G(\gamma)$ onto all of $\Gamma\left(\gamma^{\prime}\right)$ so this argument does not work for Case 3.

Lemma 3.4. The sequence

$$
E_{2, k}^{1} \rightarrow E_{1, k}^{1} \rightarrow E_{0, k}^{1} \rightarrow E_{-1, k}^{1}
$$

is exact for $A X$ when $g \geqq 4 k+1$ and for $B X$ when $g \geqq 4 k$.

Proof. This is exactly the same as the proof of 3.3 with adjustments to $g$.

Now we move to the proof of injectivity in the remaining three cases. 
Case 2. Looking at $\left(*_{3}\right)$ for $A X$, let $v[0], v[1]$ be the representatives of the vertex orbits and $e_{i}\left[\delta_{1}, \delta_{2}\right]$ the representatives of the edge orbits. Here $0 \leqq \delta_{1}, \delta_{2} \leqq 1$ are the $Q$ values of the loops, $e_{1}$ corresponds to (1 2 (2) (3 4 ), $e_{2}$ to $\left(\begin{array}{lll}1 & 3\end{array}\right)\left(\begin{array}{ll}2 & 4\end{array}\right)$ and $e_{3}$ to (1 4) (2 3). For $e_{1}$ let $\Delta$ be the 2-cell corresponding to $\left(\left(\begin{array}{lll}1 & 3\end{array}\right)\left(\begin{array}{lll}2 & 5\end{array}\right)\left(\begin{array}{ll}4 & 6\end{array}\right),\left(\delta_{1}, 0, \delta_{2}\right)\right)$. Then $\partial(\Delta)=-e_{1}\left[\delta_{1}, \delta_{2}\right]+e_{2}$ terms. Furthermore,

$$
H_{k}\left(G(\gamma)_{\Delta}\right) \rightarrow H_{k}\left(G(\gamma)_{e_{1}}\right)
$$

is surjective when $g-2 \geqq 4 k-1$ by Cases 1 and 3 of Part 1 . A similar argument holds for $e_{3}$ using (14)(26)(35). Also $e_{2}[0,0]$ and $e_{2}[1,1]$ clearly map to 0 .

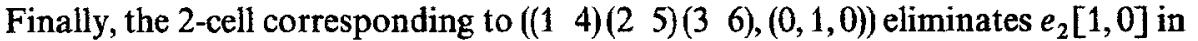
terms of $e_{2}[0,1]$ and $e_{2}[0,0]$ when $g-1 \geqq 4 k-1$. Hence

(\#)

$$
H_{k}\left(G(\gamma)_{e_{2}[0,1]}\right) \rightarrow H_{k}\left(G(\gamma)_{v[0]}\right) \oplus H_{k}\left(G(\gamma)_{v[1]}\right) \rightarrow H_{k}(G(\gamma)) \rightarrow 0
$$

is exact. But Case 1 applies to show that

$$
H_{k}\left(G(\gamma)_{e_{2}[0,1]}\right) \rightarrow H_{k}\left(G(\gamma)_{v[0]}\right)
$$

is an isomorphism. This gives Case 2.

Case 3. Using $\left(*_{3}\right)$ for $B X$, let $w[0], w[0]$ be vertex representatives and $f_{i}\left[\delta_{1}, \delta_{2}\right]$ edge ones where $0 \leqq \delta_{1}, \delta_{2} \leqq 1, f_{1}$ corresponds to the identity permutation and $f_{2}$ to (1 2). Then

$$
H_{k}\left(G(\gamma)_{f_{1}[0,1]}\right) \rightarrow H_{k}\left(G(\gamma)_{w[i]}\right)
$$

is an isomorphism for each $i$ when $g \geqq 4 k+1$ by Case 2. The face for ( $(23)$, $\left.\left(0, \delta_{1}, \delta_{2}\right)\right)$ eliminates $f_{2}\left[\delta_{1}, \delta_{2}\right]$ in terms of $f_{1}$ when $g-1 \geqq 4 k-1$. The faces for (identity, $(0, \delta, \delta)$ ) eliminate $f_{1}[\delta, \delta], \delta=0$ or 1 , when $g-1 \geqq 4 k$ and the face (identity, $(0,1,0)$ ) eliminates $f_{1}[1,0]$ in terms of $f_{1}[0,1], g-1 \geqq 4 k$. This means that

$$
H_{k}\left(G(\gamma)_{f_{1}[0,1]}\right) \rightarrow H_{k}\left(G(\gamma)_{w[0]}\right) \oplus H_{k}\left(G(\gamma)_{w[1]}\right) \rightarrow H_{k}(G(\gamma)) \rightarrow 0
$$

is exact, establishing Case 3.

Case 4. Finally, using $\left(*_{3}\right)$ for $A X$ again we have the exact sequence (\#) of Case 2. Case 3 tells us that

$$
H_{k}\left(G(\gamma)_{e_{2}[0,1]}\right) \rightarrow H_{k}\left(G(\gamma)_{\nu[1]}\right)
$$

is an isomorphism for $g-1 \geqq 4 k+2$. Case 4 follows.

\section{The case of a closed surface}

Let $G_{g, 1}$ be the stabilizer of the quadratic form $Q$ on $V=H_{1}\left(F_{g, 1} ; \mathbb{Z} / 2 \mathbb{Z}\right)$. The map $v: F_{g, 1} \rightarrow F_{g}$ obtained by attaching a disk to $\partial F_{g, 1}$ induces an isomorphism of $V$ with $V_{0}=H_{1}\left(F_{g} ; \mathbb{Z} / 2 \mathbb{Z}\right)$ so $Q$ may also be regarded as a quadratic form on $V_{0}$. Let $G_{g}$ be its stabilizer in $\Gamma_{g}$; $v$ also induces $v_{z}: G_{g, 1} \rightarrow G_{g}$ by extending eàch diffeomorphism to the identity on the attached disk. In this section we will prove:

Theorem 4.1. The map $v_{*}: H_{k}\left(G_{g, 1}\right) \rightarrow H_{k}\left(G_{g}\right)$ induced by $v_{\sharp}$ is surjective when $g \geqq 4 k+3$ and an isomorphism when $g \geqq 4 k+7$. 
Combined with Theorem 3.1 this implies that the rational homology of $G_{g}$ is independent of $g$ and $Q$ for $g$ large.

Suppose that $C$ is a nonseparating simple closed curve in $F^{0}=F_{g, 0}$ with $Q(C)=\delta$ and let $\alpha$ be an arc contained in $C$. Form $F^{1}$ by splitting $F^{0}$ open along $C$ and $F$ by splitting $F^{0}$ open only along $C-\alpha$. Then $F$ is genus $g$ with one boundary component and $F^{1}$ is obtained from $F$ by splitting along $\alpha$. We will prove that

$$
\lambda_{*}: H_{k}\left(G_{g, 1}(\alpha)\right) \rightarrow H_{k}\left(G_{g}\right)
$$

induced by the map $\lambda: F^{1} \rightarrow F^{0}$ which sews the boundary components of $F^{1}$ back together is a surjection/isomorphism for $k$ in the ranges of 4.1. The theorem will then follow from Theorem 3.1 since $\lambda_{*}=v_{*} \cdot i_{*}$ where $i: G_{g, 1}(\alpha) \hookrightarrow G_{g, 1}$ is the inclusion.

For convenience write $G^{0}=G_{g}$ and $G^{1}=G_{g, 1}(\alpha)$. Spaces $K^{i}=K\left(G^{i}, 1\right)$ can be constructed so that $K^{1} C K^{0}$ and the inclusion of $K^{1}$ into $K^{0}$ induces the map $\lambda_{*}$. Define $H_{k}\left(G^{0}, G^{1}\right)$ to be $H_{k}\left(K^{0}, K^{1}\right)$. Using the long exact sequence of the pair we see that Theorem 4.1 is equivalent to the statement that

$$
H_{k}\left(G^{0}, G^{1}\right)=0, \quad g \geqq 4 k+3 .
$$

Let $X^{i}$ be the curve system complex of $F^{i}, i=0,1$. The group $G^{i}$ acts on $X^{i}$ and $\lambda$ induces an inclusion of $X^{\mathbf{1}}$ in $X^{0}$ compatible with the actions. Furthermore, for $p \leqq g-2 \lambda$ gives a bijection between the orbits of $p$-cells under the actions of $G^{i}$ on $X^{i}$. It follows from Lemma 1.1c that there is a relative, augmented spectral sequence $\left(E^{*}, d^{*}\right)$ converging to 0 for $p+q \leqq g-3$ whose $E^{1}$ term is:

$$
E_{p, q}^{1}=\underset{\sigma_{p}}{\oplus} H_{q}\left(G_{\sigma_{p}}^{0}, G_{\sigma_{p}}^{1} ; \mathbb{Z}_{\sigma_{p}}\right), \quad p \geqq 0,
$$

with

$$
E_{-1, q}^{1}=H_{q}\left(G^{0}, G^{1}\right) .
$$

Here, in contrast to the $A X$ and $B X$ cases, there exist elements of $G^{i}$ which fix a cell of $X^{i}$ setwise but not pointwise. Therefore homology is with $\mathbb{Z}_{\sigma_{p}}$ coefficients, that is to say $\mathbb{Z}$ coefficients twisted by the orientation character $\chi_{\sigma_{p}}: G_{\sigma_{p}}^{i} \rightarrow \mathbb{Z} / 2 \mathbb{Z}$ (see [K.B.]). Note that $\chi_{\sigma_{p}}$ does not depend on $i$. Since $g \geqq 4 k+3$ implies that $g \geqq p$ $+4 q+3$ whenever $p+q \leqq k$, Theorem 4.1 now reduces to:

Lemma 4.2. $H_{q}\left(G_{\sigma_{p}}^{0}, G_{\sigma_{p}}^{1} ; \mathbb{Z}_{\sigma_{p}}\right)=0$ for $p \geqq 0$ and $g \geqq p+4 q+3$.

Let $\sigma_{p}$ correspond to the curve system $\mathscr{C}=\left\langle C_{0}, \ldots, C_{p}\right\rangle$. Then $G_{\sigma_{p}}^{i}$ is all elements of $G^{i}$ which fix $\mathscr{C}$ setwise. Define $\hat{G}_{\sigma_{p}}^{i}$ to be the subgroup of all elements which fix $\mathscr{C}$ pointwise. Then there is a short exact sequence

$$
1 \rightarrow \hat{G}_{\sigma_{p}}^{i} \rightarrow G_{\sigma_{p}}^{i} \rightarrow S\left(\sigma_{p}\right) \rightarrow 1,
$$

where $S\left(\sigma_{p}\right)=Q_{N(C)} \times Q_{p+1-N(C)}$ with $Q_{k}$ the group of signed permutations of $k$ letters. (Here the sign corresponds to the fact that each $C_{i}$ may have its orientation reversed and the permutation groups come about because elements of $G_{\sigma_{p}}^{i}$ may interchange any two curves of $\mathscr{C}$ which have the same $Q$ value.) Since $S\left(\sigma_{p}\right)$ does not depend on $i$, and $\chi_{\sigma_{p}} \mid \hat{G}_{\sigma_{p}}^{i}=1$, Lemma 4.2 reduces to the same statement with $G_{\sigma_{p}}^{i}$ replaced by $\hat{G}_{\sigma_{p}}^{i}$ and $\mathbb{Z}_{\sigma_{p}}$ replaced by $\mathbb{Z}$. 
To go further, consider the surfaces $F_{\mathscr{C}}^{i}$ obtained by splitting $F^{i}$ along $\mathscr{C}$. As we did when we passed from $F^{0}$ to $F^{1}$ we may think of $F_{\&}^{i}$ as obtained by first opening up an $\operatorname{arc} C_{j}-\alpha_{j}$ of each $C_{j}$ in $F^{i}$ to obtain a surface $\tilde{F}^{i}$ with $p+1+2 i$ boundary components and then splitting along the $p+1$ arcs $\left\{\alpha_{j}\right\}$. Reglueing each $C_{j}-\alpha_{j}$ gives $\widetilde{F}^{i} \rightarrow F^{i}$ and induces

$$
\Gamma\left(\tilde{F}^{i}\right) \rightarrow \Gamma\left(F^{i}\right) .
$$

Let $G\left(\tilde{F}^{i}\right)$ be the inverse image of $G^{i}$ and let $M_{\sigma_{p}}^{i}$ be the stabilizer of $\left\{\alpha_{j}\right\}$ in $G\left(\widetilde{F}^{i}\right)$. Then $M_{\sigma_{p}}^{i}$ may be easily identified as one of the groups $G(\gamma)$ of the previous sections. There is an exact sequence

$$
1 \rightarrow \mathbb{Z}^{p+1} \rightarrow M_{\sigma_{p}}^{i} \rightarrow \hat{G}_{\sigma_{p}}^{i} \rightarrow 1 .
$$

Here $\mathbb{Z}^{p+1}$ is generated by

$$
\left\{\tau_{C_{j}^{+}}^{2-Q\left(C_{j}\right)} \cdot \tau_{C_{j}^{-}}^{Q\left(C_{j}\right)-2}\right\}
$$

where $C_{j}$ splits into $C_{j}^{+}$and $C_{j}^{-}$in $\partial F_{C}^{i}$ and $\tau$ denotes Dehn twist. As easy application of the Lyndon-Hochschield-Serre spectral sequence reduces Lemma 4.2 to the same statement with $G_{\sigma_{p}}^{i}$ replaced by $M_{\sigma_{p}}^{i}$ and $\mathbb{Z}_{\sigma_{p}}$ replaced by $\mathbb{Z}$.

The setup to show that $H_{q}\left(M_{\sigma_{p}}^{0}, M_{\sigma_{p}}^{1}\right)=0$ is the following. The group $M_{\sigma_{p}}^{1}$ is a subgroup of $\Gamma_{g-p-2,2 p+4}$ where the boundary curves are $C_{j}^{ \pm}$coming from the $p+1$ curves $C_{j}$, and $C^{ \pm}$coming from the curve $C$. Similarly $M_{\sigma_{p}}^{0}$ is a subgroup of $\Gamma_{g-p-1,2 p+2}$. Let $\omega$ be a simple closed curve splitting $F_{g-p-2,2 p+4}$ into two surfaces, one of which is a genus zero surface with four boundary components at the curves $\omega, C^{ \pm}$and $C_{p}^{+}$. Let $M$ be the subgroup of $M_{\sigma_{p}}^{1}$ consisting of mapping classes of diffeomorphisms which are the identity on this subsurface. Then Theorem 3.1 implies that

$$
H_{q}(M) \rightarrow H_{q}\left(M_{\sigma_{p}}^{i}\right)
$$

is an isomorphism for $g-p-2 \geqq 4 q+1$. Lemma 4.2 and Theorem 4.1 follow.

\section{References}

[E.B.] Brown, E.: The Kervaire invariant of a manifold. AMS Proc. Pure Math. 22, 65-71 (1970)

[K.B.] Brown, K.: Cohomology of groups. Berlin Heidelberg New York: Springer 1982. Graduate Texts in Mathematics, vol. 87

[C] Charney, R.: Homology stability of $\mathrm{GL}_{n}$ of a Dedekind domain. Invent. Math. 56, 1-17 (1980)

[H 1] Harer, J.: The second homology group of the mapping class group of an orientable surface. Invent. Math. 72, 221-239 (1982)

[H 2] Harer, J.: Stability of the homology of the mapping class groups of orientable surfaces. Ann. Math. 121, 215-249 (1985)

[H 3] Harer, J.: The third homology group of the moduli space of curves (preprint, 1988)

[H 4] Harer, J.: The Picard group of the moduli space of curves with spin structure (preprint, 1988)

[LMW] Lee, R., Miller, E., Weintraub, S.: Rochlin invariants, theta functions and the holonomy of some determinant line bundles. J. reine angew. Math. 392, 187-218 (1988) 
[Q] Quillen, D.: MIT lectures (1974-1975)

[RS] Rourke, C., Sullivan, D.: On the Kervaire obstruction. Ann. Math. 94, 397-413 (1971)

[V] Vogtmann, K.: Spherical posets and homology stability for $\boldsymbol{O}_{n, n}$. Topology 20, 119 132 (1981)

[W] Wagoner, J.B.: Stability for homology of the general linear group of a local ring. Topology 15, 417-423 (1976)

Received January 3, 1989; in revised form November 22, 1989 